A CASE OF BLASTOMYCETIC DERMATITIS(?) JOHN GLENDON SHELDON, M.D.

Surgeon-in-Chief to the Montrose Hospital; Consulting Surgeon to the Telluride Hospital.

MONTROSE, COLO.

A Polish woman, 32 years of age, came under my care in May, 1901, suffering from a disease involving the face and causing blindness of the right eye, destruction of the nose and marked eversion of the lips. Her family history was negative. She was born in Poland and came to New York when she was 10 years old and was never sick, excepting the present illness. Venereal diseases were denied.

The history of the present complaint is as follows: About one year ago the patient noticed a pinhead-sized, firm, slightly tender swelling near the right angle of the lower jaw. The swelling was located in the skin and was freely movable. In a few days it developed into an abscess which ruptured, leaving a small ulcer. From this beginning the process extended, usually by induration and swelling followed by pus formation, crustation and, in some places, by resolution and cicatrization. In a few months the regions of the neck, mouth and nose were involved. Later the right side of the face, right eye and right ear became affected. The process still progressed, and when I first saw her the disease had advanced to the extent shown in the photograph-involving the neck, face, ears, forehead and, in places, the scalp.

The symptoms complained of were local pain, tenderness and itching: difficulty in eating and speaking, due to involvement of the lips; uncontrollable escape of salivary secretion from the mouth; epiphoria, and, during the last three weeks, progressive dimness of vision of the right eye. No other symptoms were complained of. Her general health seemed good. She felt strong. was able to work and had lost no weight. Examination of the heart, lungs, abdomen, extremities, nervous system and genitals revealed nothing abnormal. The lymphatic system was normal except that there was enlargement of the submaxillary and cervical glands. The urine was normal. Examination of the blood showed: Red corpuscles; 4,500,000; white corpuscles, 14,800; hemoglobin, 80 per cent. (sp. gt. method). Nothing abnormal in shape or size of the red cells was observed. The increase in the white cells seemed to be of the polymorphonuclear variety. The eosinophiles comprised less than 2 per cent. of the whi'e corpuscles.

The borders of the disease are everywhere well defined. In some places apparently perfectly normal skin is in contact with a smooth, thin and atrophic integumentdoubtless healed areas which had been the seats of superficial involvement of the disease. In other placeswhere deep scar formation has occurred-the skin is, more or less, wrinkled and the line between the diseased and healthy tissues is less definitely made out. The borders of the disease, corresponding to the areas, the seat of progressive involvement, are not so clearly defined as are the borders of the healed areas, the skin being thickened, reddened and indurated. In some places the diseased areas are markedly elevated. On the right side of the neck a smooth, atrophic area is elevated about $5 \mathrm{~mm}$. above the apparently healthy skin, which is continuous with it. In the median line of the neck is an area with an elevated border, covered with papillary processes which, in places, overlap the normal integument.

Examination of areas, the seat of progressive involvement of the disease, shows papillomatous growths, abscesses, crusts and ulcerated areas. Microscopically, the papillomatous growths seem to be processes of the corium covered with epithelium. Many cocci and pus corpuscles are found in the abscess cavities. The corium is vascular, infiltrated with leucocytes and round cells, and small abscesses are found containing globular and budding bodies supposed to be yeast fungi. Very few giant cells are observed. No yeast fungi were found in preparations made from the larger abscesses found on the surfaces of the diseased areas. Many small ulcers, from two millimeters to a centimeter in diameter, are found on the right side of the face and neck. These ulcers are circular, with clean-cut borders. The floors of the ulcers are smooth and no undermining of the edges is present. It seemed probable to me that these ulcers were the result of abscess formation due to infection with the ordinary pus cocci.

'The lips are so markedly everted that the patient is unable to approximate them. The eversion appears to be caused by the contraction of scars resulting from the disease. The labial mucous membrane shows no evidence of a disease similar to that affecting the face.

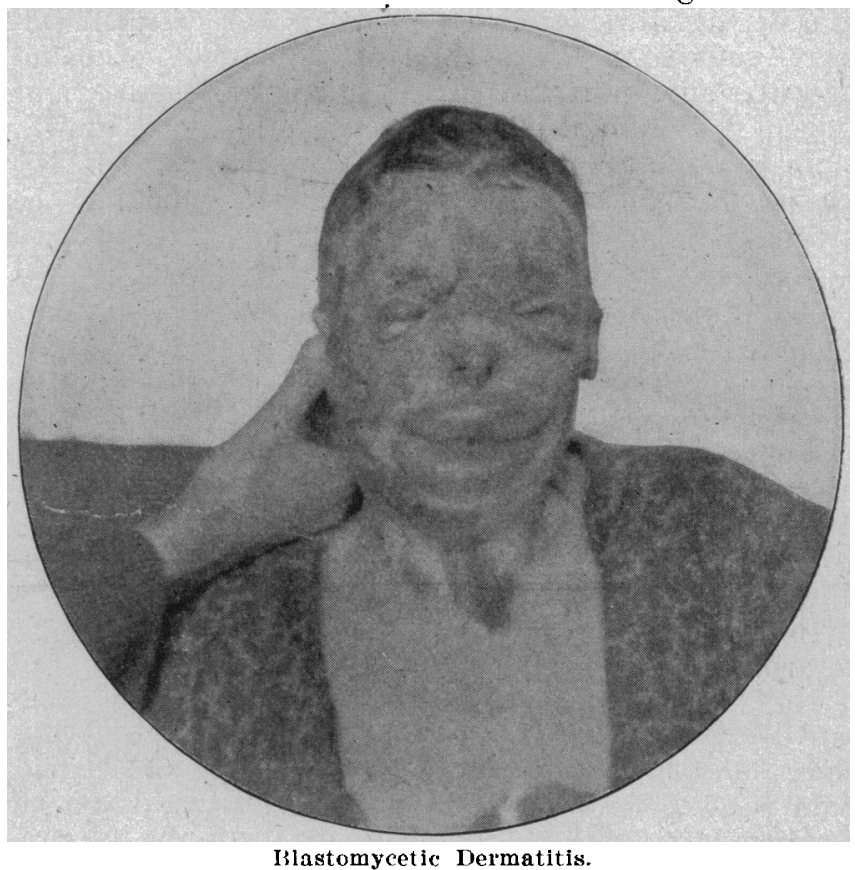

The upper and lower lids of the left eye are the seats of the disease in an active state. The conjunctiva and eyeball are not affected. The lids of the right eye present scar formation with contraction and marked eversion of the lids. The conjunctiva is involved in places, especially the upper lid and the outer part of the lower. A cicatrix is found on the inner half of the lower lid. The bulbar conjunctiva is so affected as to render the cornea opaque. No vision is present in the right eye.

The ears are affected with apparently the same process that involves the face. The scalp is involved in the right temporal and left mastoid regions. The process on the scalp seems to correspond to that on the face except that the large abscesses are more numerous and the crust formation is more abundant.

The treatment of this case was unsatisfactory. I was called out of town ten days after the patient was admitted to the hospital, and on my return found that she had left the institution, and I could not afterwards find her. During the time that the case was under my care the treatment consisted of the administration of 20 grains of potassium iodid, internally, four times a day. 
Locally, I treated five separate areas differently. One area I curetted and made no applications; another I curetted and then applied a 4 per cent. ointment of potassium iodid in vaselin; a third area was curetted and a mixture of equal parts of tincture of iodin and guaiacol applied; to the fourth area I applied pure carbolic acid, and to the fifth a mixture containing equal parts of iodin and carbolic acid. The fourth and fifth areas were not curetted.

The disease showed improvement in all of the areas treated. The area treated by curetting and applying iodin and guaiacol did better than any of the others. The least improvement was observed in the areas treated by pure carbolic acid, and by curetting followed by the potassium iodid ointment.

\section{ACUTE ANTERIOR POLIOMYELITIS IN YOUTH OF 18 YEARS. REMARKS ON THE SENSORY SYMPTOMS.}

FRANK R. FRY, A.M., M.D.

Professor of Diseases of the Nervous System, Medical Department Washington University. ST. LOUIS, MO.

A. M., aged 18 years 2 months, was taken with a febrile attack Oct. 13,1900 , accompanied by much pain in the back and thighs. and followed by acute paralysis of the lower limbs.

The patient, an intelligent young man, gives the following account of the attack: On the day he first took to bed he went to school as usual in the morning, although feeling poorly. He was too ill to remain at school, but walked home, a distance of one mile, and went to bed very ill. The next two days he was very restless, suffering especially with pain in the loins and thighs; he was so sensitive to touch that he dreaded to have anyone take hold of him, and it made him sore and tired to lie, and he was in and out of bed frequently. Finally, he could not get up and realized that his lower limbs were paralyzed. Thus, he went to bed Friday after returning from school, and on Sunday he was paralyzed.

$\mathrm{He}$ is confident that this painful stage lasted eight to ten days. He instances various collateral data which show that his statements are accurate. After the severe pain there still remained about a week during which moving and touching the paralyzed members, especially the left, caused considerable pain.

During the fourth week after going to bed he discovered that slight voluntary movements were present in the right leg, but none in the left. He did not sit up until the sixth week and then his back was so weak that he could not remain up long.

During the fever stage it was necessary to use a catheter for a day or so, and the bowels were constipated.

Dr. C. A. Anthony, Fredericktown, Mo., the attending physician, observed the case with much interest, remembering the details very accurately for the reason that they were very confusing to him for some days. His account of the onset and the great amount of hyperesthesia and pain in the back and paralyzed members conformed exactly to the statements previously obtained from the patient. No sensory tests were made at the time.

I first examined the patient Jan. 25, 1901, more than three months after the attack. I found a paraplegia of the lower extremities, very little movement in either of them, the right some better and improving faster than the left; but even the right leg had to be lifted in or out of bed or over the left. He could only get to a chair from the bed and back again with effectual assistance from a strong person.

Dr. M. W. Hoge kindly assisted me in making routine sensory and electric tests. We found all the muscular groups of both extremities more or less atrophied. The most intense atrophy was in the left thigh; apparently, the best preserved group was the calf of the right leg. The flexors of the left knee and foot were becoming contractured, and there was also a slight contracturing tendency apparent in the toes of the right foot. Electrical tests showed a reaction of degeneration, or a partial reaction of degeneration, in all the atrophied groups, the right calf and some of the right thigh muscles alone giving normal reaction. The skin of the feet and legs was cold, clammy, livid; improving in appearance from below upward and better on the right side than on the left. Careful sensory tests (touch, pressure, pain, temperature, etc.), showed slight obtundity, but not more than would be accounted for by the bad trophic state of the skin. There were no dissociate sensory symptoms, except possible pain and temperature sense were a trifle more dulled in the feet than tactile. The general sensation was improved from below upward, its impairment corresponding exactly with the nutritive impairment of the skin. There was no muscle tenderness or hyperesthesia anywhere.

This patient presents another interesting pathologie condition, which probably has no etiological relation to the myelitis, but which we may record as follows: March 31,1899 , he was accidentally shot through the chest, the ball entering to the left of the sternum about the third rib and coming out behind the right clavicle. There is an aneurysm of the arch of the aorta as a result of this injury which causes him no annoyance. He made a rapid recovery after the accident and seemed perfectly well and strong until the time of the paralytic attack which was eighteen months later.

At this writing, eight months after the attack, the patient is hobbling about on crutches, the right leg having improved considerably but the left very slightly.

On account of this patient's age there was an unusual onportunity to obtain an account of the subjective sensory symptoms in a severe attack of acute anterior poliomyelitis. I believe, as do others, that the importance of the sensory symptoms has been somewhat overlooked, and more especially in some of the text-book discussions of the disease. Without attempting an extended consideration of the question I simply wish at the present time to embrace this opportunity of calling attention to it.

Dana" thus defines the disease: "Anterior poliomyelitis is a disease of the spinal cord characterized by motor paralysis of rajid onset, followed by muscular wasting, without sensory swmptoms. It occurs at all ages but vastly oftener in infancy; hence it is often called infantile palsy."

And Starr" thus: "Anterior poliomyelitis is an acute disease observed most commonly among children, but occasionally in adults, characterized by sudden complete loss of power in one or more limbs, followed by wasting of the muscles paralyzed, and by interference of the growth of the parts, but not attended with any sensory disturbances."

Below are quotations from a number of writers whose texts I have picked from my shelves at random:

Hirt: ${ }^{3}$ Vague pains in the limbs.

Struempell: ${ }^{4}$ 'The child complains of headache and some times of pain in the loins and limbs. 